\section{Path-Following for Non-Minimum Phase Systems Removes Performance Limitations}

\author{
A. Pedro Aguiar, João P. Hespanha, and Petar V. Kokotović
}

\begin{abstract}
We highlight an essential difference between path-following and reference-tracking for non-minimum phase systems. It is well-known that in the reference-tracking, for non-minimum phase systems, there exists a fundamental performance limitation in terms of a lower bound on the $\mathcal{L}_{2}$-norm of the tracking error, even when the control effort is free. We show that this is not the case for the less stringent path-following problem, where the control objective is to force the output to follow a geometric path without a timing law assigned to it. Furthermore, the same is true even when an additional desired speed assignment is imposed.
\end{abstract}

Index Terms - Non-minimum phase systems, path-following, cheapcontrol, reference-tracking

\section{INTRODUCTION}

Obstacles to achieving perfect tracking with feedback systems have been well understood and quantified with classical Bode integrals and as the limits of cheap optimal control performance [1]-[5]. In the absence of unstable zero dynamics (non-minimum phase zeros) perfect tracking of any reference signal is possible, that is, the $\mathcal{L}_{2}$-norm of the tracking error can be made arbitrarily small. With unstable zero dynamics this is no longer possible, because an amount of "output energy" must be used for stabilization. For linear systems the tracking error increases as the signal frequencies approach those of the unstable zeros [4], [6].

The limitations introduced by unstable zero dynamics are structural. They cannot be avoided without changing the system structure or re-formulating the tracking problem. One such reformulation is to select a new output for which the zero dynamics are stable and perfect tracking is possible [7], [8]. As discussed in [8], the new output should be physically meaningful and allow a good approximation to the original tracking task.

Another reformulation of the tracking problem investigated in this note is to divide it into two tasks: geometric path following and speed assignment along the path. As shown in [9], this two-task formulation is suitable for many applications. It also offers the flexibility to use the timing law as an additional control variable and thus change the input/output structure of the system. In particular, this flexibility can be employed for a rescaling of the exosystem eigenvalues in the internal model approach.

The main contribution of this note is to present conditions under which for linear non-minimum phase systems the two-task path following problem can be solved with arbitrarily small $\mathcal{L}_{2}$-norm of the path following error. In Section II we formulate the path-following problems. After a brief review of reference-tracking in Section III, Section IV presents the main results of the note showing that the well-known performance limitations imposed on reference-tracking by non-minimum phase zeros do not appear in the less stringent pathfollowing problem. An example in Section V illustrates the results. Concluding remarks are given in Section VI.

The work of A. Pedro Aguiar was supported in part by a Pos-Doc Fellowship PRAXIS XXI from the Portuguese Foundation of Science and Technology. The work of J. P. Hespanha and P. V. Kokotović were supported by the National Science Foundation under Grants ECS-0242798 and ECS-0228846, respectively. The authors are with the Center for Control Engineering and Computation, University of California, Santa Barbara, CA 93106-9560 USA (e-mail: aguiar@ece.ucsb.edu; hespanha@ece.ucsb.edu; petar@ece.ucsb.edu).
Notation: Transpose is denoted by ', complex conjugate by *, and complex conjugate transpose by ${ }^{H}$. The complex plane is denoted by $\mathbb{C}=\mathbb{C}^{-} \cup \mathbb{C}^{0} \cup \mathbb{C}^{+}$, where $\mathbb{C}^{-}:=\{s \in \mathbb{C}: \operatorname{Re}(s)<0\}$, $\mathbb{C}^{0}:=\{s \in \mathbb{C}: \operatorname{Re}(s)=0\}$, and $\mathbb{C}^{+}:=\{s \in \mathbb{C}: \operatorname{Re}(s)>0\}, \operatorname{Re}$ is the real part and Im is the imaginary part. The Laplace transform of $x(t)$ is $X(s) . \mathcal{L}_{2}$ is the space of square integrable vector functions over $\left[t_{0}, \infty\right)$ with norm $\int_{t_{0}}^{\infty}\|\cdot\|^{2} d t$.

\section{PATH-FOLLOWING PROBlems}

Path-following problems are primarily concerned with the design of control laws that drive an object (robot arm, mobile robot, ship, aircraft, etc.) to reach and follow a geometric path. A secondary goal is to force the object moving along the path to satisfy some additional dynamic specification. A common approach to the pathfollowing problem is to parameterize the geometric path $y_{d}$ by a path variable $\theta$ and then select a timing law for $\theta$, [9]-[13]. Extending the approach of [10], a path-following controller was proposed in [9] for a class of uncertain strict feedback nonlinear systems. A framework for path-following as a method to avoid some limitations in reference-tracking was described in [13]. The key idea is to use $\theta$ as an additional control input to stabilize the unstable zero-dynamics while the original control variables keep the system on the path.

In this note, we consider geometric path-following for nonminimum phase systems and paths formed by a linear combination of sinusoids in $\theta$. When the control effort is free, we show that there is no limitation upon the achievable path error performance. The same result holds even when we impose an additional speed assignment $v_{d}$ for the path variable $\theta(t)$.

This essential difference between reference-tracking and pathfollowing shows that is not appropriate to re-formulate a pathfollowing problem as a reference-tracking by making $\theta(t)=v_{d} t$. In many applications this would introduce limits on the achievable performance that are not inherent to the original problem.

We now proceed with the problem formulation. For the linear timeinvariant system

$$
\begin{aligned}
& \dot{x}(t)=A x(t)+B u(t), \quad x\left(t_{0}\right)=x_{0} \\
& y(t)=C x(t)+D u(t),
\end{aligned}
$$

where $x(t) \in \mathbb{R}^{n}$ is the state, and $u(t) \in \mathbb{R}^{m}$ the control, the output $y(t) \in \mathbb{R}^{q}, m \geq q$, is required to reach and follow a geometric path

$$
y_{d}(\theta):=\sum_{k=1}^{n_{d}}\left[a_{k} e^{j \omega_{k} \theta}+a_{k}^{\star} e^{-j \omega_{k} \theta}\right],
$$

where $\theta$ is the scalar path parameter, $\omega_{k}>0$ are real numbers, and $a_{k}$ are non-zero complex vectors.

The geometric path $y_{d}(\theta)$ can be generated by an exosystem of the form

$$
\begin{aligned}
\frac{d}{d \theta} w(\theta) & =S w(\theta), \quad w\left(\theta_{0}\right)=w_{0} \\
y_{d}(\theta) & =Q w(\theta),
\end{aligned}
$$

where $w \in \mathbb{R}^{2 n_{d} q}$ is the exogenous state and $S+S^{\prime}=0$. For any timing law $\theta(t)$, the path-following error is defined as

$$
e(t):=y(t)-y_{d}(\theta(t)) .
$$

The two path-following problems to be solved are as follows. 


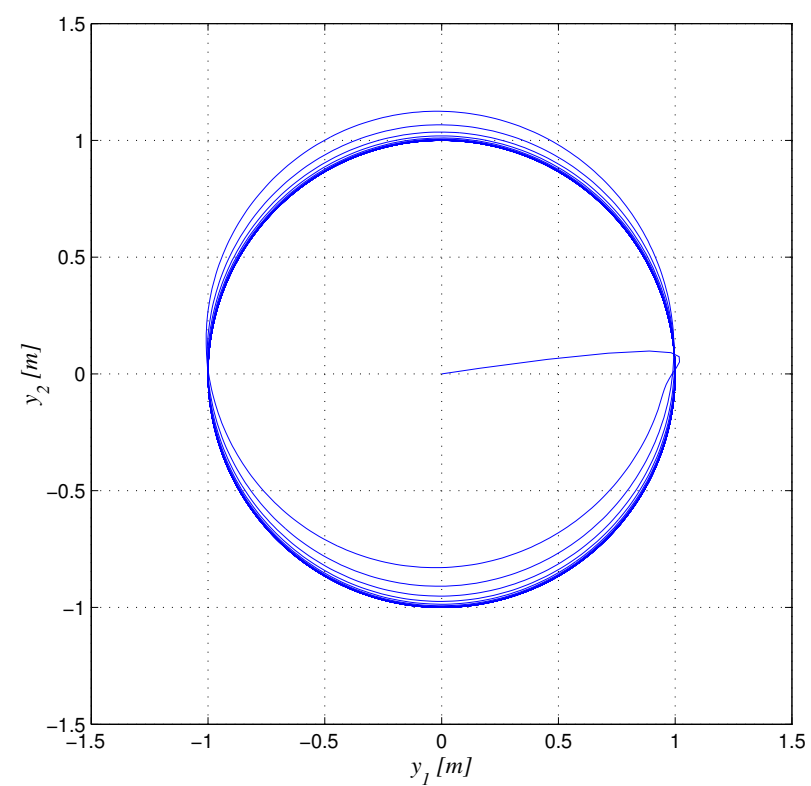

(a)

Fig. 1. Trajectory of the vehicle: 1(a) reference-tracking, 1(b) path-following

Geometric path-following: For a desired path $y_{d}(\theta)$, design a controller that achieves:

i) boundedness: the state $x(t)$ is uniformly bounded for all $t \geq t_{0}$ and every initial condition $\left(x\left(t_{0}\right), w\left(\theta_{0}\right)\right), \theta_{0}:=\theta\left(t_{0}\right)$,

ii) error convergence: the path-following error $e(t)$ converges to zero as $t \rightarrow \infty$, and

iii) forward motion: $\dot{\theta}(t)>c$ for all $t \geq t_{0}$, where $c$ is a positive constant.

Speed-assigned path-following: In addition to geometric pathfollowing, given a desired speed $v_{d}>0$, it is required that either $\dot{\theta}(t) \rightarrow v_{d}$ as $t \rightarrow \infty$, or $\dot{\theta}(t)=v_{d}$ for $t \geq T$ and some $T \geq t_{0}$.

As illustrated by Skjetne et al. [9], these path-following problems provide natural settings for many engineering applications, including situations when the timing law $\theta(t)$ is determined by a human operator. From a theoretical standpoint our main interest is to determine whether the freedom to select a timing law $\theta(t)$ can be used to achieve an arbitrarily small $\mathcal{L}_{2}$-norm of the path-following error, that is, whether $\delta>0$ in

$$
J:=\int_{t_{0}}^{\infty}\|e(t)\|^{2} d t \leq \delta
$$

can be made arbitrarily small.

Before we address this question in Section IV, let us briefly recall that for the standard reference-tracking the answer is, in general, negative.

\section{REVIEW OF REFERENCE-TRACKING}

The standard linear reference-tracking problem is to design a feedback controller for (1) such that the closed-loop state is bounded, and for any reference signal $r(t)$, the output $y(t)$ asymptotically approaches $r(t)$. For $r(t)$ generated by a known exosystem,

$$
\dot{w}(t)=S w(t), \quad r(t)=Q w(t),
$$

the so-called regulator or servomechanism problem, was shown in [1], [14]-[16], to be solvable if and only if $(A, B)$ is stabilizable,

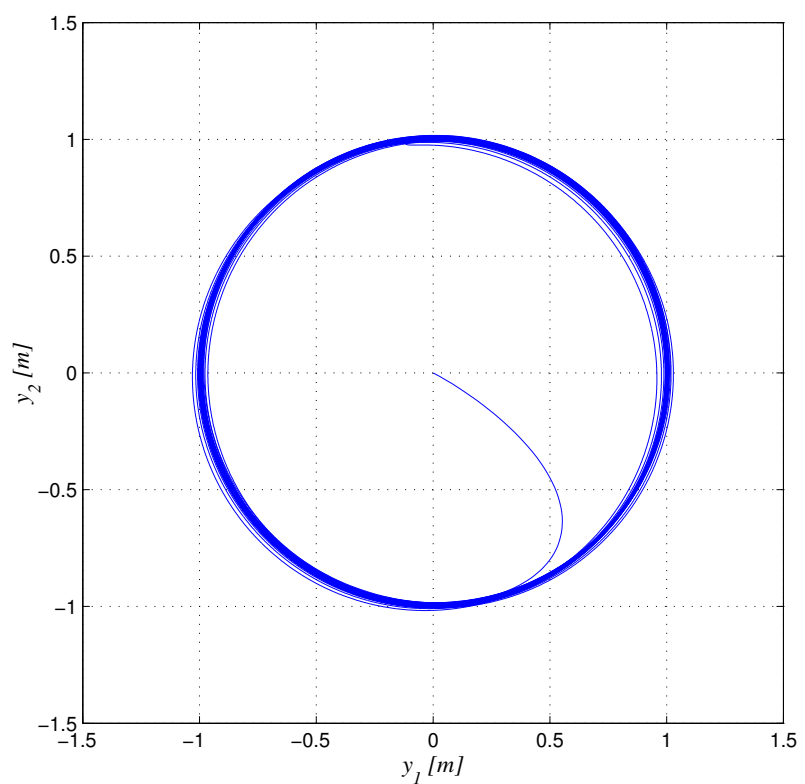

(b)
$(C, A)$ is detectable, the number of inputs is at least as large as the number of outputs $(m \geq q)$ and the zeros of $(A, B, C, D)$ do not coincide with the eigenvalues of $S$.

In that case, the internal model approach of Francis [15], [17] uses matrices $\Pi$ and $\Gamma$ that satisfy

$$
\begin{aligned}
\Pi S & =A \Pi+B \Gamma, \\
0 & =C \Pi+D \Gamma-Q,
\end{aligned}
$$

to design the reference-tracking controller

$$
u(t)=K x(t)+(\Gamma-K \Pi) w(t),
$$

where $K$ is such that $(A+B K)$ is Hurwitz. With this controller, the transients $\tilde{x}:=x-\Pi w$ and $\tilde{u}:=u-\Gamma w$ converge to zero and are governed by $\dot{\tilde{x}}=(A+B K) \tilde{x}, \tilde{u}=K \tilde{x}$.

An important issue in reference-tracking problems is whether the $\mathcal{L}_{2}$-norm of the tracking error can be made arbitrarily small, that is,

$$
\int_{t_{0}}^{\infty}\|y(t)-r(t)\|^{2} d t \leq \delta
$$

can be satisfied for any $\delta>0$. For this to be the case, the zeros of $(A, B, C, D)$ must be in the open left half-plane $\mathbb{C}^{-}$.

The non-minimum phase zeros, that is the zeros in $\mathbb{C}^{+}$, impose a fundamental limitation on the attainable tracking performance $J$. This is revealed by the fact that the limit as $\epsilon \rightarrow 0$ of the optimal value of the cost functional

$$
J_{\epsilon}=\min _{\tilde{u}} \int_{t_{0}}^{\infty}\left[\|y(t)-r(t)\|^{2}+\epsilon^{2}\|\tilde{u}(t)\|^{2}\right] d t
$$

is strictly positive, [1]. Qiu and Davison [4] showed that for $x\left(t_{0}\right)=$ $0, r(t)=\eta_{1} \sin \omega t+\eta_{2} \cos \omega t$, the best attainable performance is

$$
\begin{gathered}
J=\eta^{\prime} M \eta, \quad \eta=\operatorname{col}\left(\eta_{1}, \eta_{2}\right) \\
\operatorname{trace} M=\sum_{i=1}^{p}\left(\frac{1}{z_{i}-j \omega}+\frac{1}{z_{i}+j \omega}\right),
\end{gathered}
$$

where $M \geq 0$, and $z_{1}, z_{2}, \ldots, z_{p}$ are the non-minimum zeros of $(A, B, C, D)$. With $\omega=0$ the same formula holds for $r(t)=$ const. 
For step reference signals and multivariable linear systems, Chen, Qiu, and Toker [18] show that the effect of nonminimum phase zeros is determined not only by the zero locations, but also the mutual orientation between zero and input signal directions. For more general reference signals, Su, Qiu, and Chen [6] give explicit formulas which show the dependence of $J$ on the non-minimum phase zeros and their frequency-dependent directional information.

Seron et al. [5] re-interpreted the Qiu-Davison formula [4] and generalized it to a class of nonlinear systems. They showed that the best attainable value of $J$ is equal to the lowest control effort, measured by the $\mathcal{L}_{2}$-norm, needed to stabilize the zero dynamics driven by the system output $y(t)$. It is its role as a stabilizing control input that prevents the output $y(t)$ from perfect tracking. Extensions to non-right-invertible systems are given in [19], [20]. Control energy constraints as another source of fundamental performance limitations have been investigated in [21].

\section{MAIN RESUlts}

We now show that the attainable performance for the pathfollowing problems is not limited by non-minimum phase zeros. As in [4], [6], [19], [20], we assume that initially the system is at rest, that is, we let $x\left(t_{0}\right)=0$.

Theorem 1: If $(A, B)$ is stabilizable, then for the geometric pathfollowing problem and any given positive constant $\delta$ there exist constant matrices $K$ and $L$, and a timing law $\theta(t)$ such that the feedback law

$$
u(t)=K x(t)+L w(\theta(t))
$$

achieves (5)

Proof: Using (8) and the timing law

$$
\dot{\theta}(t)=v_{d}, \quad \theta\left(t_{0}\right)=\theta_{0}
$$

where $v_{d}>0$ is a constant to be selected, we can view the pathfollowing problem as the reference-tracking of the signal

$$
\begin{aligned}
r(t) & =y_{d}(\theta(t))=\sum_{k=1}^{n_{d}}\left[a_{k} e^{j \omega_{k} \theta(t)}+a_{k}^{\star} e^{-j \omega_{k} \theta(t)}\right] \\
& =\sum_{k=1}^{n_{d}}\left[a_{k_{0}} e^{j v_{d} \omega_{k}\left(t-t_{0}\right)}+a_{k_{0}}^{\star} e^{-j v_{d} \omega_{k}\left(t-t_{0}\right)}\right],
\end{aligned}
$$

where $a_{k_{0}}:=a_{k} e^{j \omega_{k} \theta_{0}}$. From the exosystem (3) and (9) we obtain the set of equations

$$
\begin{aligned}
\dot{x}(t) & =A x(t)+B u(t), \\
\dot{w}(t) & =v_{d} S w(t), \\
e(t) & =C x(t)-Q w(t),
\end{aligned}
$$

that describe a regulator problem to which the results of [15], [22], [23] are applicable. The constant $v_{d}$ should be selected such that the zeros of (1) do not coincide with the eigenvalues of $v_{d} S$.

To prove that for any $\delta>0$, we can select $K, L$, and $v_{d}$ such that (5) holds, we apply the results in [4], [6] to (10b)-(11) and obtain

$$
\begin{gathered}
J=\eta^{\prime} M \eta \leq\|\eta\|^{2} \operatorname{trace} M, \\
\operatorname{trace} M=\sum_{i=1}^{p} \sum_{k=1}^{n_{d}}\left(\frac{1}{z_{i}-j v_{d} \omega_{k}}+\frac{1}{z_{i}+j v_{d} \omega_{k}}\right),
\end{gathered}
$$

where $z_{1}, z_{2}, \ldots, z_{p}$ are the zeros of $(A, B, C, D)$ in $\mathbb{C}^{+}$and

$$
\begin{aligned}
\eta:=2 \operatorname{col}\left(\operatorname{Re} a_{1_{0}}, \operatorname{Im} a_{1_{0}}, \operatorname{Re} a_{2_{0}}, \operatorname{Im} a_{2_{0}},\right. \\
\left.\cdots, \operatorname{Re} a_{n_{d 0}}, \operatorname{Im} a_{n_{d 0}}\right) .
\end{aligned}
$$

This is shown in Appendix and will also be useful in the proof of Theorem 2. The result follows from (12) because for any $\delta>0$ there is a sufficiently large $v_{d}$ such that (5) holds.

Remark 1: We stress that the stabilizability of $(A, B)$ is the only condition (necessary and sufficient) for the solvability of the geometric path-following problem using (8).

Next we show that an arbitrarily small $\mathcal{L}_{2}$-norm of the pathfollowing error is attainable even when the speed assignment $v_{d}$ is specified beforehand.

Theorem 2: Let $v_{d}$ be specified so that the eigenvalues of $v_{d} S$ do not coincide with the zeros of (1), and assume that $(A, B)$ is stabilizable. Then, for the speed-assigned path-following problem, (5) can be satisfied for any $\delta>0$ with a timing law $\theta(t)$ and a controller of the form (8) but with time-varying piecewise-constant matrices $K$ and $L$.

Proof: To construct a path-following controller that satisfies (5) we start with the following structure

$$
\begin{aligned}
& u=K_{\sigma}\left(x-\Pi_{\sigma} w\right)+\Gamma_{\sigma} w, \\
& \dot{\theta}=v_{\sigma},
\end{aligned}
$$

where for each positive constant $v_{\ell}, \ell \in \mathcal{I}$, the matrices $\Pi_{\ell}$ and $\Gamma_{\ell}$ satisfy

$$
\begin{aligned}
v_{\ell} \Pi_{\ell} S & =A \Pi_{\ell}+B \Gamma_{\ell}, \\
0 & =C \Pi_{\ell}+D \Gamma_{\ell}-Q,
\end{aligned}
$$

and $\sigma(t):\left[t_{0}, \infty\right) \rightarrow \mathcal{I}, \mathcal{I}:=\{0,1,2, \ldots N\}$ is the piecewise constant switching signal

$$
\sigma(t)= \begin{cases}0, & t_{0} \leq t<t_{1} \\ 1, & t_{1} \leq t<t_{2} \\ \vdots & \\ N, & t \geq t_{N}\end{cases}
$$

Each $K_{\ell}$ is chosen such that the matrix $\left(A+B K_{\ell}\right)$ is Hurwitz. The existence of matrices $\Pi_{\ell}$ and $\Gamma_{\ell}$ presumes that $v_{\ell}$ will be chosen so that the eigenvalues of $v_{\ell} S$ do not coincide with the zeros of (1). We observe that (14) is a speed-assignment path-following controller for which $\dot{\theta}(t)$ converges to $v_{N}=v_{d}$ in finite time.

We now prove that any given performance specification $\delta$, (5) can be satisfied by appropriate selection of the finite sequence $t_{0}, t_{1}, \ldots, t_{N}$ together with the parameters $\left(v_{0}, \Pi_{0}, \Gamma_{0}, K_{0}\right)$,

$\left(v_{1}, \Pi_{1}, \Gamma_{1}, K_{1}\right), \ldots,\left(v_{N}, \Pi_{N}, \Gamma_{N}, K_{N}\right)$ used in the feedback controller (14). We start by selecting $v_{0}$ sufficiently large such that defining

$$
\bar{J}_{0}:=\|\eta\|^{2} \sum_{i=1}^{p} \sum_{k=1}^{n_{d}}\left(\frac{1}{z_{i}-j v_{0} \omega_{k}}+\frac{1}{z_{i}+j v_{0} \omega_{k}}\right),
$$

where $z_{1}, z_{2}, \ldots, z_{p}$ are the zeros of $(A, B, C, D)$ in $\mathbb{C}^{+}$and $\eta$ is defined by (13), $\bar{J}_{0}$ is upper-bounded by $\frac{\delta}{4}$. We show in Appendix that $J$ is upper-bounded by

$$
\begin{aligned}
J \leq & \bar{J}_{0}+\sum_{\ell=1}^{N} \gamma\left(v_{\ell-1}-v_{\ell}\right)^{2}\|\eta\|^{2} \\
& +2 \sum_{\ell=1}^{N} \tilde{x}_{\ell-1}^{i n}\left(t_{\ell}\right)^{\prime}\left(\bar{x}_{\ell-1}^{i n}\left(t_{\ell}\right)-\bar{x}_{\ell}^{i n}\left(t_{\ell}\right)\right) \\
& +\sum_{\ell=1}^{N}\left\|\tilde{x}_{\ell-1}^{i n}\left(t_{\ell}\right)\right\|^{2},
\end{aligned}
$$

where $\gamma$ is a positive constant, $\bar{x}_{\ell}^{i n}:=F_{\ell} w$, with the $F_{\ell}$ constant matrices of appropriate dimensions, and the transient state errors $\tilde{x}_{\ell}^{i n}(t)$ converge to zero as fast as $e^{\left(A+B K_{\ell}\right)}$. 
We show that each term of (17) can be upper-bounded by $\frac{\delta}{4}$ so that $J \leq \delta$. The first term is smaller than $\frac{\delta}{4}$ by construction. To prove that the second term in (17) is also smaller than $\frac{\delta}{4}$, we select the parameters $v_{\ell}, \ell \in \mathcal{I}$ to satisfy

$$
v_{\ell-1}-v_{\ell}=\mu, \quad v_{N}=v_{d}, \quad \ell=1,2, \ldots, N
$$

where $\mu:=\delta /\left(4 \gamma\|\eta\|^{2}\left(v_{0}-v_{N}\right)\right)$, and $N:=\frac{v_{0}-v_{N}}{\mu}$. Then, it follows that the second term in (17) can be upper-bounded by

$$
\begin{aligned}
\sum_{\ell=1}^{N} \gamma\left(v_{\ell-1}-v_{\ell}\right)^{2}\|\eta\|^{2} & \leq N \gamma\|\eta\|^{2} \mu^{2} \\
& =\left(v_{0}-v_{N}\right) \gamma\|\eta\|^{2} \mu=\frac{\delta}{4}
\end{aligned}
$$

The above selection for the $v_{\ell}, \ell \in \mathcal{I}$, is made under the constraint that the eigenvalues of $v_{\ell} S$ do not coincide with the zeros of (1). This can always be satisfied by appropriately adjusting $v_{0}$. Finally, for a given finite $N$, each of the last two terms in (17) can be made smaller than $\frac{\delta}{4}$ by choosing $t_{\ell}, \ell=1,2, \ldots, N$ sufficiently large.

\section{ILLUSTRATIVE EXAMPLE}

A vehicle with mass $M$ moving in the plane, on top of which lies a mass $m$, is modeled by

$$
M \ddot{y}=D(\dot{z}-\dot{y})+u, \quad m \ddot{z}=D(\dot{y}-\dot{z})+G(z-y),
$$

where $D=\operatorname{diag}\left(d_{1}, d_{2}\right)>0, G=\operatorname{diag}\left(g_{1}, g_{2}\right)>0, u \in \mathbb{R}^{2}$ is the force, $y \in \mathbb{R}^{2}$ and $z \in \mathbb{R}^{2}$ are the positions of the vehicle and the mass $m$, respectively. The mass $m$ is "carried" by the vehicle via the viscous friction force $D(\dot{y}-\dot{z})$, while the gravity force $G(z-y)$ drives the mass away from the position $z=y$ because the top of the vehicle is not flat. The system is non-minimum phase with two zeros in $\mathbb{C}^{+}$located at $z_{i}=\left(\sqrt{d_{i}^{2}+4 m g_{i}}-d_{i}\right) /(2 m), i=1,2$.

The path-following task is to move the vehicle along a circular path with the desired steady-state velocity $v_{d}=1$. The path is centered at the origin with radius $R=1$. The vehicle starts with zero initial conditions and the model parameters are $M=1, m=0.1, D=$ $\operatorname{diag}(15,10), G=\operatorname{diag}(1.5,1)$.

For the sake of comparison, we first recast this problem as a reference-tracking problem by creating the reference signal

$$
r(t)=\operatorname{col}\left(R \cos \left(v_{d}\left(t-t_{0}\right)\right), R \sin \left(v_{d}\left(t-t_{0}\right)\right)\right), \quad \forall t \geq t_{0}
$$

Fig. 1(a) displays the simulation results obtained with the control gain $K$ computed by solving the optimal cheap control problem (7) with $\epsilon=0.001$. As expected, the convergence to the desired trajectory is achieved with a significant transient error: $J \simeq 0.23$.

In contrast, Fig. 1(b) shows the simulation results obtained with the path-following controller described in the proof of Theorem 2. Starting with $v_{0}=10$, the values of $v_{\ell}$ were selected to decrease by 0.5 in 10 seconds to $v_{N}=v_{d}=1(N=18)$ at time $t=t_{N}=180 \mathrm{~s}$. The values of the control gains $K_{\ell}, \ell \in \mathcal{I}$, are all equal to the value of $K$ used in the first experiment. As it can be seen, the convergence of the vehicle to the path is much smoother and the transient error is reduced to $J \simeq 0.06$.

\section{CONCLUSions}

We have revisited the classical issue of performance limitations in reference-tracking for linear non-minimum phase systems. We have demonstrated that the performance limitations can be avoided by reformulating the problem as path-following, where the path variable $\theta$ is treated as an additional control variable. This conceptual result may be of practical significance, because the path-following formulation is convenient for many applications. Design of pathfollowing controllers for non-minimum phase systems and the study of performance under control energy constraints are topics for future research.

\section{APPENDIX}

To derive (12) and inequality (17), we will need the following results:

- A transfer matrix $\mathcal{P}_{i n}(s)$ is said to be inner if $\mathcal{P}_{i n}(s)$ is stable with all the zeros located in $\mathbb{C}^{+}$and $\mathcal{P}_{i n}^{\prime}(-s) \mathcal{P}_{i n}(s)=I \forall s \in$ $\mathbb{C}$. A transfer matrix $\mathcal{P}_{\text {out }}(s)$ is said to be outer if it has full row rank in the open right half complex plane. Every transfer matrix $\mathcal{P}(s)$ can be factored as $\mathcal{P}(s)=\mathcal{P}_{\text {in }}(s) \mathcal{P}_{\text {out }}(s)$ such that $\mathcal{P}_{\text {in }}(s)$ is inner and $\mathcal{P}_{\text {out }}(s)$ is outer [4, Lemma 2].

- A minimal realization $(A, B, C, D)$ of a stable transfer matrix $\mathcal{P}$ is called a balanced realization if the solutions $P, Q$ to the Lyapunov equations

$$
\begin{aligned}
& A P+P A^{\prime}=-B B^{\prime} \\
& A^{\prime} Q+Q A=-C^{\prime} C
\end{aligned}
$$

are diagonal and equal.

\section{A. Derivation of (12)}

This derivation closely follows [4]. Since $\bar{x}=\Pi w, \bar{u}=\Gamma w$, the transients $\tilde{x}=x-\bar{x}, \tilde{u}=u-\bar{u}$ satisfy

$$
\begin{aligned}
\dot{\tilde{x}} & =A \tilde{x}+B \tilde{u}, \quad \tilde{x}\left(t_{0}\right)=-\bar{x}\left(t_{0}\right), \\
e & =C \tilde{x}, \\
\tilde{u} & =K \tilde{x} .
\end{aligned}
$$

We need to compute the limit $J=\lim _{\epsilon \downarrow 0} J_{\epsilon}$ of the cheap optimal control problem

$$
J_{\epsilon}=\min _{\tilde{u}} \int_{t_{0}}^{\infty}\left[e(t)^{\prime} e(t)+\epsilon^{2} \tilde{u}(t)^{\prime} \tilde{u}(t)\right] d t, \quad \epsilon>0
$$

for (1) with zero initial conditions. The optimal control law is $\tilde{u}=K_{\epsilon} \tilde{x}$, where $K_{\epsilon}=-\frac{1}{\epsilon^{2}} B^{\prime} P_{\epsilon}$, and $P_{\epsilon}$ is the unique, positive semidefinite solution of the algebraic Riccati equation

$$
A^{\prime} P_{\epsilon}+P_{\epsilon} A+C^{\prime} C=\frac{1}{\epsilon^{2}} P_{\epsilon} B B^{\prime} P_{\epsilon} .
$$

It is well known [1] that $P_{0}=\lim _{\epsilon \downarrow 0} P_{\epsilon}$ exists, and is independent of which stabilizable and detectable state-space realizations the plant is used. As in [4] and without loss of generality, we let $(A, B, C, D)$ be consistent with the inner-outer factorization $\mathcal{P}(s)=\mathcal{P}_{\text {in }}(s) \mathcal{P}_{\text {out }}(s)$, where $\mathcal{P}(s)$ is the transfer matrix of system $(1), \mathcal{P}_{i n}(s)$ is a square stable inner transfer matrix and $\mathcal{P}_{\text {out }}(s)$ is a minimum phase rightinvertible transfer matrix. Let $\left(A_{i n}, B_{i n}, C_{i n}, D_{i n}\right)$ be a balanced realization of $\mathcal{P}_{\text {in }}(s)$ and $\left(A_{\text {out }}, B_{\text {out }}, C_{\text {out }}, D_{\text {out }}\right)$ be any stabilizable and detectable realization of $\mathcal{P}_{\text {out }}(s)$. The partitioning of $x$ as $x=\operatorname{col}\left(x^{\text {in }}, x^{\text {out }}\right)$ gives

$$
\begin{aligned}
& A=\left[\begin{array}{cc}
A_{\text {in }} & B_{\text {in }} C_{\text {out }} \\
0 & A_{\text {out }}
\end{array}\right], \\
& C=\left[\begin{array}{cc}
C_{\text {in }} & D_{\text {in }} C_{\text {out }}
\end{array}\right], \quad D=\left[\begin{array}{c}
B_{\text {in }} D_{\text {out }} \\
B_{\text {out }}
\end{array}\right], \\
& D_{\text {in }} D_{\text {out }},
\end{aligned}
$$


and from Theorem 1 in [4] it follows that $P_{0}=\lim _{\epsilon \rightarrow 0} P_{\epsilon}=\left[\begin{array}{ll}I & 0 \\ 0 & 0\end{array}\right]$, and hence

$$
J=\lim _{\epsilon \rightarrow 0} J_{\epsilon}=\tilde{x}^{i n}\left(t_{0}\right)^{\prime} \tilde{x}^{i n}\left(t_{0}\right)=\bar{x}^{i n}\left(t_{0}\right)^{\prime} \bar{x}^{i n}\left(t_{0}\right)
$$

since $\tilde{x}^{i n}\left(t_{0}\right)=-\bar{x}^{i n}\left(t_{0}\right)$. The Laplace transform of $\bar{x}^{i n}(t)$ and $\bar{y}(t)$ are

$$
\begin{aligned}
\bar{X}^{\text {in }}(s) & =\left(s I-A_{\text {in }}\right)^{-1} B_{\text {in }} \bar{Y}^{\text {out }}(s), \\
\bar{Y}(s) & =\mathcal{P}_{\text {in }}(s) \bar{Y}^{\text {out }}(s) .
\end{aligned}
$$

Therefore,

$$
\bar{X}^{i n}(s)=L(s) \bar{Y}(s), \quad L(s)=\left(s I-A_{i n}\right)^{-1} B_{i n} \mathcal{P}_{i n}^{-1}(s) .
$$

Since $\bar{y}=y_{d}$, the substitution of $y_{d}(t)$ from (10a) gives

$$
\begin{aligned}
& \bar{x}^{i n}(t)=\sum_{k=1}^{n_{d}}\left[L\left(j v_{d} \omega_{k}\right) a_{k} e^{j \omega_{k} \theta(t)}\right. \\
& \left.+L\left(-j v_{d} \omega_{k}\right) a_{k}^{\star} e^{-j \omega_{k} \theta(t)}\right] \\
& =2 \sum_{k=1}^{n_{d}}\left[\operatorname{Re} L\left(j v_{d} \omega_{k}\right) \operatorname{Re} a_{k_{0}}\right. \\
& \left.-\operatorname{Im} L\left(j v_{d} \omega_{k}\right) \operatorname{Im} a_{k_{0}}\right] .
\end{aligned}
$$

The computation of $J=\bar{x}^{i n}\left(t_{0}\right)^{\prime} x^{i n}\left(t_{0}\right)$ results in $J=\eta^{\prime} M \eta$ where $M$ is shown at the bottom of the page, and $\eta$ is given in (13). Finally, the formula (12) is obtained by observing that trace $M=\sum_{k=1}^{n_{d}} \operatorname{trace}\left[L^{H}\left(j v_{d} \omega_{k}\right) L\left(j v_{d} \omega_{k}\right)\right], \mathcal{P}_{i}$ is a inner matrix, and $B_{i} B_{i}^{\prime}=-A_{i}-A_{i}^{\prime}$, [4].

\section{B. Derivation of (17)}

We first compute

$$
J_{\ell}:=\int_{t_{\ell}}^{\infty} e(t)^{\prime} e(t) d t, \quad \ell \in \mathcal{I}
$$

with $\sigma(t)=\ell$ for all $t \geq t_{\ell}$ and note that

$$
J \leq \sum_{\ell=0}^{N} J_{\ell}
$$

As in the derivation of formula (12), we get

$$
J_{\ell}=\tilde{x}_{\ell}^{i n}\left(t_{\ell}\right)^{\prime} \tilde{x}_{\ell}^{i n}\left(t_{\ell}\right)
$$

where $\tilde{x}_{\ell}:=x-\bar{x}_{\ell}$ is partitioned as $\tilde{x}_{\ell}=\operatorname{col}\left(\tilde{x}_{\ell}^{i n}, \tilde{x}_{\ell}^{\text {out }}\right)$, and $\bar{x}_{\ell}=\Pi_{\ell} w$, that is, $\bar{x}_{\ell}$ is the steady-state of $x$ when $\sigma(t)=\ell$ for all $t \geq t_{\ell}$. For $\ell=0, J_{0}$ is given by (12) replacing $v_{d}$ by $v_{0}$, and satisfies $J_{0} \leq \bar{J}_{0}$, where $\bar{J}_{0}$ is defined in (16). For $\ell=1,2, \ldots, N$ we substitute $x\left(t_{\ell}\right)=\tilde{x}_{\ell-1}\left(t_{\ell}\right)+\bar{x}_{\ell-1}\left(t_{\ell}\right)$ in (24) and get

$$
\begin{aligned}
J_{\ell}= & \left(\bar{x}_{\ell-1}^{i n}\left(t_{\ell}\right)+\tilde{x}_{\ell-1}^{i n}\left(t_{\ell}\right)-\bar{x}_{\ell}^{i n}\left(t_{\ell}\right)\right)^{\prime} \\
& \times\left(\bar{x}_{\ell-1}^{i n}\left(t_{\ell}\right)+\tilde{x}_{\ell-1}^{i n}\left(t_{\ell}\right)-\bar{x}_{\ell}^{i n}\left(t_{\ell}\right)\right) \\
= & \left(\bar{x}_{\ell-1}^{i n}\left(t_{\ell}\right)-\bar{x}_{\ell}^{i n}\left(t_{\ell}\right)\right)^{\prime}\left(\bar{x}_{\ell-1}^{i n}\left(t_{\ell}\right)-\bar{x}_{\ell}^{i n}\left(t_{\ell}\right)\right) \\
& +2 \tilde{x}_{\ell-1}^{i n}\left(t_{\ell}\right)^{\prime}\left(\bar{x}_{\ell-1}^{i n}\left(t_{\ell}\right)-\bar{x}_{\ell}^{i n}\left(t_{\ell}\right)\right) \\
& +\tilde{x}_{\ell-1}^{i n}\left(t_{\ell}\right)^{\prime} \tilde{x}_{\ell-1}^{i n}\left(t_{\ell}\right) .
\end{aligned}
$$

With $\theta(t)=v_{\ell-1}\left(t-t_{\ell-1}\right)$ substituted in (2) and using (22a) we obtain

$$
\begin{aligned}
\bar{x}_{\ell-1}^{i n}\left(t_{\ell}\right)-\bar{x}_{\ell}^{i n}\left(t_{\ell}\right)=\sum_{k=1}^{n_{d}}[ & L\left(j v_{\ell-1} \omega_{k}\right) a_{k} e^{j \omega_{k} \theta\left(t_{\ell}\right)} \\
& +L\left(-j v_{\ell-1} \omega_{k}\right) a_{k}^{\star} e^{-j \omega_{k} \theta\left(t_{\ell}\right)} \\
& -L\left(j v_{\ell} \omega_{k}\right) a_{k} e^{j \omega_{k} \theta\left(t_{\ell}\right)} \\
& \left.+L\left(-j v_{\ell} \omega_{k}\right) a_{k}^{\star} e^{-j \omega_{k} \theta\left(t_{\ell}\right)}\right] \\
=2 \sum_{k=1}^{n_{d}}[ & \left(\operatorname{Re} L\left(j v_{\ell-1} \omega_{k}\right)\right. \\
& \left.-\operatorname{Re} L\left(j v_{\ell} \omega_{k}\right)\right) \operatorname{Re} a_{k_{\ell}} \\
& -\left(\operatorname{Im} L\left(j v_{\ell-1} \omega_{k}\right)\right. \\
& \left.\left.-\operatorname{Im} L\left(j v_{\ell} \omega_{k}\right)\right) \operatorname{Im} a_{k_{\ell}}\right]
\end{aligned}
$$

where $a_{k_{\ell}}:=a_{k} e^{j \omega_{k} \theta_{\ell}}, \ell \in \mathcal{I}, k=1,2, \ldots, n_{d}$, and $\theta_{\ell}:=\theta\left(t_{\ell}\right)$, $\ell \in \mathcal{I}$. Since $\mathcal{P}_{i}(s)$ has no poles on the imaginary axis, $\operatorname{Re} L\left(j v_{\ell} \omega_{k}\right)$ and $\operatorname{Im} L\left(j v_{\ell} \omega_{k}\right)$ are continuously differentiable functions of $v_{\ell}$, and by the mean value theorem, it follows that the equation shown at the top of the next page holds, where $A_{\ell, k}, B_{\ell, k}, k=1, \ldots, n_{d}$, $\ell=1, \ldots N$ are constant matrices and the vector $\eta$ is defined in (13). Therefore,

$$
\begin{aligned}
J_{\ell}=\eta^{\prime} M_{\ell} \eta\left(v_{\ell-1}-v_{\ell}\right)^{2}+2 \tilde{x}_{\ell-1_{i}}\left(t_{\ell}\right)^{\prime} \\
\left(\bar{x}_{\ell-1}^{i n}\left(t_{\ell}\right)-\bar{x}_{\ell}^{i n}\left(t_{\ell}\right)\right)+\tilde{x}_{\ell-1_{i}}\left(t_{\ell}\right)^{\prime} \tilde{x}_{\ell-1_{i}}\left(t_{\ell}\right)
\end{aligned}
$$

where

$$
M_{\ell}:=\left[\begin{array}{c}
A_{\ell, 1}^{\prime} \\
-B_{\ell, 1}^{\prime} \\
\vdots \\
A_{\ell, n_{d}}^{\prime} \\
-B_{\ell, n_{d}}^{\prime}
\end{array}\right]\left[\begin{array}{llll}
A_{\ell, 1}-B_{\ell, 1} & \cdots & A_{\ell, n_{d}}-B_{\ell, n_{d}}
\end{array}\right]
$$

Then (17) follows from (23), (25), and the fact that there exists a sufficiently large $\gamma>0$ such that $\operatorname{trace}\left(M_{\ell}\right) \leq \gamma$ for any $v_{\ell} \in$ $\left[v_{d}, v_{0}\right]$.

$$
M:=\left[\begin{array}{c}
\operatorname{Re}^{\prime} L\left(j v_{d} \omega_{1}\right) \\
-\operatorname{Im}^{\prime} L\left(j v_{d} \omega_{1}\right) \\
\vdots \\
\operatorname{Re}^{\prime} L\left(j v_{d} \omega_{n_{d}}\right) \\
-\operatorname{Im}^{\prime} L\left(j v_{d} \omega_{n_{d}}\right)
\end{array}\right]\left[\operatorname{Re} L\left(j v_{d} \omega_{1}\right),-\operatorname{Im} L\left(j v_{d} \omega_{1}\right), \cdots, \operatorname{Re} L\left(j v_{d} \omega_{n_{d}}\right),-\operatorname{Im} L\left(j v_{d} \omega_{n_{d}}\right)\right],
$$




$$
\begin{aligned}
\bar{x}_{\ell-1}^{i n}\left(t_{\ell}\right)-\bar{x}_{\ell}^{i n}\left(t_{\ell}\right) & =2 \sum_{k=1}^{n_{d}}\left[A_{\ell, k}\left(v_{\ell-1}-v_{\ell}\right) \operatorname{Re} a_{k_{\ell-1}}-B_{\ell, k}\left(v_{\ell-1}-v_{\ell}\right) \operatorname{Im} a_{k_{\ell-1}}\right] \\
& =\left[A_{\ell, 1}-B_{\ell, 1} \cdots A_{\ell, n_{d}}-B_{\ell, n_{d}}\right] \eta\left(v_{\ell-1}-v_{\ell}\right),
\end{aligned}
$$

\section{REFERENCES}

[1] H. Kwakernaak and R. Sivan, "The maximal achievable accuracy of linear optimal regulators and linear optimal filters," IEEE Trans. on Automat. Contr., vol. 17, no. 1, pp. 79-86, Feb. 1972.

[2] B. Francis, "The optimal linear-quadratic time-invariant regulator with cheap control," IEEE Trans. on Automat. Contr., vol. 24, no. 4, pp. 616621, Aug. 1979.

[3] R. H. Middleton, "Trade-offs in linear control systems design," Automatica, vol. 27, no. 2, pp. 281-292, Mar. 1991.

[4] L. Qiu and E. J. Davison, "Performance limitations of nonminimum phase systems in the servomechanism problem," Automatica, vol. 29, pp. 337-349, 1993.

[5] M. M. Seron, J. H. Braslavsky, P. V. Kokotović, and D. Q. Mayne, "Feedback limitations in nonlinear systems: From bode integrals to cheap control," IEEE Trans. on Automat. Contr., vol. 44, no. 4, pp. 829-833, Apr. 1999.

[6] W. Su, L. Qiu, and J. Chen, "Fundamental performance limitations in tracking sinusoidal signals," IEEE Trans. on Automat. Contr., vol. 48, no. 8, pp. 1371-1380, Aug. 2003.

[7] M. Fliess, H. Sira-Ramírez, and R. Marquez, "Regulation of nonminimum phase outputs: a flatness based approach." in Perspectives in Control-Theory and Applications: a Tribute to Ioan Doré Landau, D. Normand-Cyrot, Ed. London: Springer, 1998, pp. 143-164.

[8] M. Fliess and R. Marquez, "Continuous-time linear predictive control and flatness: a module-theoretic setting with examples," Int. J. of Control, vol. 73, no. 7, pp. 606-623, 2000.

[9] R. Skjetne, T. I. Fossen, and P. Kokotović, "Robust output maneuvering for a class of nonlinear systems," Automatica, vol. 40, no. 3, pp. 373383, 2004.

[10] J. Hauser and R. Hindman, "Maneuver regulation from trajectory tracking: Feedback linearizable systems," in Proc. IFAC Symp. Nonlinear Control Systems Design, Lake Tahoe, CA, USA, 1995, pp. 595-600.

[11] S. Al-Hiddabi and N. McClamroch, "Tracking and maneuver regulation control for nonlinear nonminimum phase systems: application to flight control," IEEE Trans. on Contr. Systems Tech., vol. 10, no. 6, pp. 780792, 2002.

[12] A. P. Aguiar and J. P. Hespanha, "Logic-based switching control for trajectory-tracking and path-following of underactuated autonomous vehicles with parametric modeling uncertainty," in Proc. of the 2004 Amer. Contr. Conf., Boston, MA, USA, June 2004.

[13] A. P. Aguiar, D. B. Dačić, J. P. Hespanha, and P. Kokotović, "Pathfollowing or reference-tracking? An answer relaxing the limits to performance," in Proc. of IAV2004 - 5th IFAC/EURON Symp. on Intel. Auton. Vehicles, Lisbon, Portugal, July 2004.

[14] E. J. Davison, "The robust control of a servomechanism problem for linear time-invariant multivariable systems," IEEE Trans. on Automat. Contr., vol. 21, no. 1, pp. 25-34, Feb. 1976.

[15] B. Francis, "The linear multivariable regulator problem," SIAM J. Contr. Optimization, vol. 15, no. 3, pp. 486-505, May 1977.

[16] E. J. Davison and B. Scherzinger, "Perfect control of the robust servomechanism problem," IEEE Trans. on Automat. Contr., vol. 32, no. 8, pp. 689-702, Aug. 1987.

[17] B. Francis and W. Wonham, "The internal model principle of control theory," Automatica, vol. 12, no. 5, pp. 457-465, 1976.

[18] J. Chen, L. Qiu, and O. Toker, "Limitations on maximal tracking accuracy," IEEE Trans. on Automat. Contr., vol. 45, no. 2, pp. 326-331, Feb. 2000.

[19] A. Woodyatt, M. Seron, J. Freudenberg, and R. Middleton, "Cheap control tracking performance for non-right-invertible systems," Int. J. of Robust and Nonlinear Control, vol. 12, no. 15, pp. 1253-1273, Dec. 2002.

[20] J. H. Braslavsky, R. Middleton, and J. Freudenberg, "Cheap control performance of a class of nonright-invertible nonlinear systems," IEEE Trans. on Automat. Contr., vol. 47, no. 8, pp. 1314-1319, Aug. 2002.

[21] J. Chen, S. Hara, and G. Chen, "Best tracking and regulation performance under control energy constraint," IEEE Trans. on Automat. Contr., vol. 48, no. 8, pp. 1320-1336, Aug. 2003.
[22] M. Hautus, Linear matrix equations with applications to the regulator problem, I. D. Landau ed., ser. Outils and Modéles Mathématique pour l'Automatique, Paris: C.N.R.S., 1983, pp. 399-412.

[23] A. Isidori and C. I. Byrnes, "Output regulation of nonlinear systems," IEEE Trans. on Automat. Contr., vol. 35, no. 2, pp. 131-140, Feb. 1990. 